$\mathcal{G S}_{\text {http://dx.doi.org/10.3765/sp.5.1 }}^{\text {Semantics \& Pragmatics Volume 5, Article 1: 1-25, } 2012}$

\title{
Two types of class B numeral modifiers: A reply to Nouwen 2010*
}

\author{
Bernhard Schwarz \\ McGill University
}

\author{
Brian Buccola \\ McGill University
}

Michael Hamilton
McGill University

Received 2011-07-29 / First Decision 2011-09-12 / Revisions Received 2011-11-14 /

Second Decision 2011-11-21 / Published 2012-02-13

\begin{abstract}
Nouwen (2010) proposes that numeral modifiers like at most and up to belong to a natural class of expressions that share the same semantic interpretation. We identify several semantic contrasts between at most and up to that are inconsistent with this proposal. Moreover, we show that Nouwen's semantics cannot be right for at most. In attempting to apply his semantics to up to, we propose an amendment intended to accomodate certain observations not captured in Nouwen's original account.
\end{abstract}

Keywords: modified numerals, degree scales, ignorance implications, monotonicity, weak numerals

* For discussion of material related to this article, we thank the participants of three syntaxsemantics research group meetings at McGill during the summer and fall of 2011. We also thank two anonymous referees, as well as David Beaver, for comments that have helped us improve the final version. The first author was supported in part by SSHRC Standard Research Grant 410-2010-1264 (principal investigator: Junko Shimoyama) and FQRSC Programme Soutien aux Équipes de Recherche grant 2012-SE-144646 (principal investigator: Lisa Travis). The second author received support from SSHRC Standard Research Grant 410-2010-1254 (principal investigator: Brendan Gillon), and a McCall McBain Fellowship from the McGill Faculty of Arts. The third author received support from SSHRC Research Grants 410-20111062 (principal investigator: Michael Wagner) and 410-2008-1687 (principal investigator: Lisa Travis), as well as SSHRC Strategic Grant 869-2009-0004 (principal investigator: Michael Wagner).

(C2012 Bernhard Schwarz, Brian Buccola, and Michael Hamilton

This is an open-access article distributed under the terms of a Creative Commons NonCommercial License (creativecommons.org/licenses/by-nc/3.0). 
Bernhard Schwarz, Brian Buccola, and Michael Hamilton

\section{Introduction}

So-called numeral modifiers combine with numerals into expressions like less than ten, at most five, etc. The focus of Nouwen 2010 is an interesting contrast between two types of numeral modifiers, illustrated in (1) and (2).

(1) A hexagon has fewer than 11 sides.

(2) a. \#A hexagon has at most 10 sides.

b. \#A hexagon has up to 10 sides.

(Nouwen 2010: 3:3)

Nouwen attributes the acceptability contrast between the sentences in (1) and (2) to a semantic contrast between the numeral modifiers they contain. Specifically, he takes the numeral modifiers in (2) to introduce an implication of speaker ignorance as to the number of sides a hexagon has. Nouwen attributes the oddness of the particular examples in (2) to the implausibility of this implication, which (1) lacks. ${ }^{1}$

Nouwen refers to numeral modifiers that introduce ignorance implications as class $B$ modifiers and those that do not as class $A$ modifiers. Furthermore, Nouwen distinguishes upper-bound numeral modifiers, such as those in (1) and (2), from lower-bound numeral modifiers, such as more than, at least and minimally.

In this reply we focus on Nouwen's analysis of upper-bound class B numeral modifiers. Nouwen argues that all upper-bound class B numeral modifiers share the same interpretation. We present data that challenge this proposal, identifying two types of semantic contrasts between at most and up to: one involving NPI licensing and the corresponding inferences and another involving what we refer to as the bottom-of-the-scale effect. We explore how Nouwen's analysis applies to these two upper-bound class B modifiers. We argue that it fails for at most, but we explore how a conservative amendment of his analysis, extended with what we call the range requirement, applies to up to. One consequence, if our amendment is on the right track, is that the ignorance implications for at most and up to have different sources.

In section 2, we review Nouwen's analysis of upper-bound class B numeral modifiers. Section 3 identifies a contrast between at most and up to with regard to NPI licensing and the corresponding inferences. Section 4 describes

1 We take Nouwen's use of "\#” in (2) to indicate mere implausibility, as opposed to downright unacceptability. In later sections, we apply "\#” differently to mark semantic/pragmatic ill-formedness in our own examples. 
Two types of class B numeral modifiers

another contrast with regard to the bottom-of-the-scale effect, and we provide a characterization of it within the framework employed by Nouwen. In section 5 we propose and explore an amended semantics for up to which incorporates the range requirement. We show that, apart from capturing the bottomof-the-scale effect, it has the welcome consequence of deriving ignorance implications. In section 6 we identify and discuss a point of tension between our account and the existence of weak interpretations of numerals. Section 7 concludes.

\section{Nouwen's semantics for class B numeral modifiers}

\subsection{Bare numerals}

Following Hackl 2000, Nouwen (2010) proposes that bare numerals, such as three in (3), serve as the argument of a silent counting quantifier, many, as shown in the logical form or $L F$ in (4).

(3) Three students attended.

(4) [[three many] students] attended

Nouwen assigns to many the denotation in (5), where $X$ is a singular or plural individual and $|X|$ is the number of singular parts of $X{ }^{2}$

$$
\llbracket \text { many } \rrbracket=\lambda d_{d} \cdot \lambda f_{e t} \cdot \lambda g_{e t} \cdot \exists ! X[|X|=d \& f(X)=g(X)=T]
$$

Under this semantics for many, the LF in (4) will be assigned the truth conditions in (6), which states that exactly three students attended.

$$
\exists ! X[|X|=3 \& \llbracket \text { students } \rrbracket(X)=\llbracket \text { attended } \rrbracket(X)=T ~ \rrbracket
$$

\subsection{Upper-bound class B numeral modifiers and covert modality}

Following Hackl 2000, Nouwen (2010) assumes that a numeral modifier combines with a numeral to form a degree phrase denoting a generalized quantifier over degrees. For interpretability, this degree phrase must raise

2 Actually, Nouwen introduces two numeral counting quantifiers: a strong many (which he calls $m a n y_{2}$ ), with the denotation in (5), and a weak many (many $)_{1}$ ), as in Hackl 20oo. Since the weak many is not central to Nouwen's account of numeral modifiers, nor to our discussion of it, we disregard it until section 6 . 
covertly to derive a degree predicate in its scope. Under these assumptions, (8) is an interpretable LF for the example in (7).

(7) At most three students attended.

(8) [[at most] three] $\lambda d[[[d$ many] students] attended]

The lexical entry that Nouwen proposes for upper-bound class B numeral modifiers like at most and up to is shown in (9), according to which these modifiers equate the degree denoted by the numeral with the maximum element of the set given by the degree predicate.

(9) $\llbracket$ at most/up to $\rrbracket=\lambda d_{d} \cdot \lambda f_{d t} \cdot \max (f)=d$

Under this semantics for upper-bound class B numeral modifiers, the LF in (8) is assigned the truth conditions in (10).

$$
\max (\{\mathrm{d}: \exists ! \mathrm{X}[|\mathrm{X}|=\mathrm{d} \& \llbracket \text { students } \rrbracket(X)=\llbracket \text { attended } \rrbracket(X)=\mathrm{T}]\})=3
$$

The set of degrees to which max applies in (10) is the singleton set containing the cardinality of the set of students who attended, i.e., the exact number of attending students. ${ }^{3}$ Of course, the maximum element of a singleton set is its single element. Accordingly, (10), like (6), states that exactly three students attended.

To be sure, this is not an attested interpretation of (7), nor does Nouwen claim that it is. Nouwen credits the actual truth conditions of (7) to a different LF, namely the one in (11). This LF differs from (8) only in that " $\diamond$ ", a silent speaker-oriented epistemic possibility operator, appears in the immediate scope of the degree phrase.

(11) $\quad$ [[at most] three] $\lambda \mathrm{d}[\diamond[[[\mathrm{d}$ many $]$ students $]$ attended $]]$

The LF in (11) receives the truth conditions in (12), which states that the maximal number of students who could have attended, according to the speaker's evidence, is three. Nouwen suggests that this is the intended interpretation of $(7)$.

(12) $\quad \max (\{\mathrm{d}: \diamond \exists ! \mathrm{X}[|\mathrm{X}|=\mathrm{d} \& \llbracket$ students $\rrbracket(X)=\llbracket$ attended $\rrbracket(X)=\mathrm{T}]\})=3$

3 We are putting aside, for now, the problematic case where the set of students who attended is empty. We return to this issue in section 4 , where it will be important. 
Two types of class B numeral modifiers

Two questions remain. First, what excludes the unattested truth conditions in (10), hence what excludes the LF in (8)? Nouwen proposes that the exclusion of this LF is a blocking effect of the sort familiar from the morphophonological literature (e.g., Kiparsky 1973, 1983). Specifically, Nouwen takes the LF in (8) to be blocked by the truth conditionally equivalent but less marked LF in (4). ${ }^{4}$

Second, how does Nouwen's account derive the ignorance implications which he takes to be a defining feature of class B numeral modifiers? Surprisingly, this is not addressed. Note that, (12), as it stands, does not entail speaker ignorance regarding the number of students who attended. We return to this issue in section 5 .

\section{NPI licensing and downward entailment}

\subsection{At most}

It has been observed in the literature that phrases headed by at most license negative polarity items (NPIs), such as ever in (13) and give a damn in (14).

(13) At most three people had ever been in this cave.

(Krifka 2007)

(14) At most three students give a damn about Pavarotti.

(Chierchia \& McConnell-Ginet 2000: 522)

This observation puts Nouwen's (2010) analysis of upper-bound class B modifiers in conflict with Ladusaw's (1979) classic account of NPI licensing, which equates the class of NPI licensors with the class of downward entailing expressions. Under Nouwen's account, at most three is clearly not downward entailing; in fact, it is non-monotone. Obviously, for any two sets of degrees $\mathrm{A}$ and $\mathrm{B}$ such that $\mathrm{A}$ is a subset of $\mathrm{B}$, nothing guarantees that $\max (\mathrm{A})$ equals $\max (\mathrm{B})$.

Moreover, it would not be possible for Nouwen to resolve this conflict by simply rejecting Ladusaw's analysis of NPI licensing. Putting aside NPIs, intuitions on inferences alone suggest that at most three should be analyzed as downward entailing. The validity of relevant inferences, like the one from (15a) to (15b), seems to be uncontroversial. ${ }^{5}$

4 Even though Nouwen doesn't present a general theory of markedness, it indeed seems plausible to us to assume that (8) is more marked than (4).

5 Actually, in view of the ignorance implications with class B modifiers described by Nouwen (see section 1 above), the intuitive validity of this inference is not necessarily expected. To 
(15) a. At most three students smoke.

b. At most three students smoke cigars.

(Chierchia \& McConnell-Ginet 2000: 522)

And yet, by virtue of the non-monotone semantics Nouwen assigns to at most three, the satisfaction of the truth conditions of (16a), shown in (17a), does not guarantee the satisfaction of the truth conditions of (16b), shown in (17b).

a. [[at most] three] $\lambda \mathrm{d}[\diamond$ [[[d many] students] smoke $]$

b. [[at most] three] $\lambda \mathrm{d}[\diamond[[[\mathrm{d}$ many] students] [smoke cigars]]]

$$
\begin{aligned}
\text { a. } \max (\{\mathrm{d}: \diamond \exists ! X[|X|=\mathrm{d} \& \llbracket \text { students } \rrbracket(X)=\llbracket \text { smoke } \rrbracket(X)=\mathrm{T}]\})=3 \\
\text { b. } \max (\{\mathrm{d}: \diamond \exists ! X[|\mathrm{X}|=\mathrm{d} \& \llbracket \text { students } \rrbracket(X)=\llbracket \text { smoke cigars } \rrbracket(X)=\mathrm{T}]\}) \\
=3
\end{aligned}
$$

For example, in a scenario where the speaker is sure that exactly one, two or three students smoke, while also being sure that exactly one or two (but not three) students smoke cigars, (17a) is true, while (17b) is false. Thus, it is clear that Nouwen cannot account for the attested inference from (15a) to (15b).

\subsection{Up to}

Recall now that one of Nouwen's (2010) main points is that upper-bound class B modifiers, including up to and at most, share the same semantic interpretation. It turns out that up to is markedly different from at most when it comes to NPI licensing. (18) and (19) are unacceptable, unlike their counterparts with at most in (13) and (14).

(18) *Up to three people had ever been in this cave.

(19) *Up to three students give a damn about Pavarotti.

Furthermore, in accordance with Ladusaw's (1979) analysis of NPI licensing, up to fails to support the corresponding inferences. That is, our intuitions indicate that (2ob) cannot be inferred from (20a).

be sure, it is possible to be uncertain about the exact number of students who smoke while being certain about the exact number of students who smoke cigars. Apparently, ignorance implications with at most are not obligatory. Looking ahead, note that this is consistent with our working hypothesis in section 5 that ignorance implications with up to are part of the asserted, truth-conditional content. 
Two types of class B numeral modifiers

(20) a. Up to three students smoke.

b. Up to three students smoke cigars.

Specifically, the same scenario described above readily comes to mind according to our intuitions: In a scenario where the speaker is sure that exactly one, two or three students smoke, while also being sure that exactly one or two (but not three) students smoke cigars, (20a) is true and appropriate, while (2ob) is not. ${ }^{6}$

These observations cast doubt on Nouwen's proposal that all class B modifiers share the same semantic interpretation. ${ }^{7}$ However, while we have excluded the lexical entry in (9) for at most, it might be just what is needed for up to. In particular, not only does Nouwen's analysis predict the unacceptability of (18) and (19) and the invalidity of the inference from (20a) to (2ob), it also fits with our intuitions, noted above, as to why this inference is invalid. ${ }^{8}$

6 It is clear that it would be inappropriate for a speaker to utter (2ob) in the scenario provided. However, it is not so clear that (2ob) is judged to be false, even though Nouwen's analysis predicts that it is false. Under Nouwen's analysis, there is a missing link between falsity and the judgment of inappropriateness. This issue could be attributed to the fact that the sentences in question are statements about the speaker's epistemic state. The question, which we leave to future research, is how predicted truth values in such cases translate into judgments on appropriateness. Thanks to David Beaver for raising this issue.

Note also that, as predicted by the non-monotone semantics for up to, we do not judge the reverse inference from (20b) to (20a) to be valid, either. (20a) conveys information that (20b) does not, viz. that (the speaker is certain that) no more than three students smoke.

7 Citing Gajewski's (2008) analysis of only and no one but, a reviewer suggests that the NPI licensing contrast presented above could be consistent with semantic equivalence of at most and up to if it could be argued that the two differ in internal syntactic structure. The reviewer's point is well taken. However, we are not sure how to spell out this suggestion, since it appears to us that a structural contrast in the spirit of Gajewski 2008 would necessitate a semantic contrast as well. Moreover, the following section presents an additional contrast between up to and at most which we interpret as further indication that the two are not semantically equivalent.

8 As illustrated by the contrast in pragmatic well-formedness between (i) and (ii) below, there are dramatic differences in use between up to and at most.

(i) Save up to $50 \%$ on airtime rates!

(ii) \#Save at most $50 \%$ on airtime rates!

While we leave the detailed analysis of such contrasts as a topic for future work, we speculate that they relate to the monotonicity contrast described in this section. 
Bernhard Schwarz, Brian Buccola, and Michael Hamilton

\section{Bottom-of-the-scale effect}

\subsection{Another contrast}

The pattern in (21) and (22) illustrates another systematic contrast between at most and up to, showing that up to is sensitive to the choice of the numeral being modified in a way that at most is not.

(21) a. At most ten people died in the crash.

b. Up to ten people died in the crash.

(Nouwen 2008: 580)

(22) a. At most one person died in the crash.

b. \#Up to one person died in the crash.

While at most combines freely with either one or a higher numeral like ten, the use of up to in (22b) is unacceptable. A natural speculation as to the relevant difference between one and ten is that one in some sense denotes the bottom of the scale. The acceptability contrast in (22) suggests, then, that up to cannot combine with a bottom-of-the-scale numeral. We refer to this restriction on up to as the bottom-of-the-scale effect.

The observation that this effect is only attested with one of the two modifiers under consideration further questions Nouwen's (2010) proposal that class B modifiers share the same semantic interpretation. In the previous section, we concluded that Nouwen's semantics does not apply correctly to

at most, but is still an option for up to. Therefore, for the remainder of this paper we confine our attention to the latter.

It turns out that, as it stands, Nouwen's analysis of class B modifiers fails to predict the bottom-of-the-scale effect with up to. The analysis assigns sentence (22b) the LF in (23), which receives the truth conditions in (24).

(23) [[up to] one $\lambda \mathrm{d}[\diamond$ [[d many] people died in the crash]]

(24) $\max (\{\mathrm{d}: \diamond \exists ! X[|\mathrm{X}|=\mathrm{d} \& \llbracket$ people $\rrbracket(X)=\llbracket$ died in the $\operatorname{crash} \rrbracket(X)=\mathrm{T}]\})=1$

The statement in (24) amounts to saying that, according to the speaker's evidence, no more than one person could have died in the crash. Since this would seem to be a useful meaning for a sentence to convey, no explanation for the unacceptability of (22b) emerges.

To accomodate the bottom-of-the-scale effect, we now present and explore a conservative revision of Nouwen's account. 
Two types of class B numeral modifiers

\subsection{The bottom of the scale}

Naturally, to explain the bottom-of-the-scale effect, we will first have to elucidate the notion of bottom of the scale. We begin by taking a closer look at the set of degrees in (25), the set to which max applies in (24) above.

(25) $\{\mathrm{d}: \diamond \exists ! X[|\mathrm{X}|=\mathrm{d} \& \llbracket$ person $\rrbracket(X)=\llbracket$ died in the $\operatorname{crash} \rrbracket(X)=\mathrm{T}]\}$

The smallest possible element of (25) depends in part on what exactly the variable $X$ is taken to range over. Nouwen's (2010) assumptions in this regard are unclear to us. But the issue is addressed in Hackl 2000, which established the framework for Nouwen's analysis of numeral modifiers. Hackl is explicit in not assuming the existence of a zero individual, i.e., an individual corresponding to the empty set in set theory. ${ }^{9}$ The absence of such a possible value for $\mathrm{X}$ guarantees that (25) cannot have o as an element.

In fact, we can be certain that (25) does not contain any degree smaller than 1: While it is perhaps possible for half a person to be photographed or to be covered with sand, it is impossible for half a person to die in a crash. The smallest possible degree contained in the set (25) is accordingly 1, i.e., the degree that we have characterized as the bottom of the scale.

The bottom of the scale, then, is the smallest possible element of the set of degrees to which the degree phrase headed by up to applies under (our construal of) Nouwen's analysis. In more theory-neutral terms, a bottom-ofthe-scale numeral denotes the lowest non-zero degree that can satisfy the degree property provided by the sentence that contains it. So 1 is the bottom of the scale in (22b) because a fatal crash necessarily implies the death of at least one person.

This general characterization of the bottom of the scale suggests that it need not always be given by one, hence that the bottom-of-the-scale effect can be reproduced with other numerals. This is indeed so. Suppose, for example, that eggs can be bought in half-dozen cartons only. Under this assumption, our judgments on the examples (26b) and (27b) are parallel to those we gave for (21b) and (22b) above. In particular, we detect the same unacceptability in (27b) as in (22b). ${ }^{10}$

9 Hackl (2000: 169) writes: "Note that the definition does not allow us to count zero individuals, i.e., there is no individual that is assigned degree zero in a complete join semi-lattice. I will have to leave it for future research to extend the definition so that expressions like 'more than zero students', 'fewer than one students', etc. can be covered."

10 Replacing a dozen and half a dozen with twelve and six, respectively, does not significantly alter our judgments. 
(26) (Assumption: Eggs can be bought in half-dozen cartons only.)

a. He bought at most a dozen eggs.

b. He bought up to a dozen eggs.

(27) (Assumption: Eggs can be bought in half-dozen cartons only.)

a. He bought at most half a dozen eggs.

b. \#He bought up to half a dozen eggs.

Under Nouwen's analysis, (27b) has the LF in (28), which receives the truth conditions in (29); (30) isolates the set of degrees to which max applies in (29).

(28) [[up to] half a dozen] $\lambda \mathrm{d}[\diamond$ [he bought [d many] eggs]]

(29) $\max (\{\mathrm{d}: \diamond \exists ! X[|\mathrm{X}|=\mathrm{d} \& \llbracket$ eggs $\rrbracket(X)=\llbracket$ he bought $\rrbracket(X)=\mathrm{T}]\})=6$

(30) $\quad\{\mathrm{d}: \diamond \exists ! \mathrm{X}[|\mathrm{X}|=\mathrm{d} \& \llbracket$ eggs $\rrbracket(\mathrm{X})=\llbracket$ he bought $\rrbracket(\mathrm{X})=\mathrm{T}]\}$

By assumption, any purchase of eggs has to be a purchase of at least six. Accordingly, in the absence of a zero individual, 6 is the smallest possible element of the set of degrees in (30). Thus, half a dozen in (27b) denotes the bottom of the scale, and so we are not surprised that (27b) shares the oddness of (22b).

It is also possible, of course, for some scale not to have a bottom at all. In such a case, the bottom-of-the-scale effect is not expected to arise with one or with any degree-denoting phrase, for that matter. For example, since the amount of cake consumed in a cake-eating event can be arbitrarily small, and in particular less than one entire cake, one in (31) need not denote the bottom of the scale. Hence, we correctly expect this example to be acceptable. ${ }^{11}$

She ate up to one whole cake.

11 Example (31) sounds better with whole (or entire) than without, and we speculate that this is because whole signals the possibility of eating less than one cake, i.e., it signals that the relevant scale reaches below 1 . Note that this speculation is supported by the oddness of the sentence One entire person died in the crash, whose content is inconsistent with a scale reaching below 1 .

Note also that, while it seems clear that the contrast between (31) and (22b) is to be explained along the lines suggested here, the framework that Nouwen adopts from Hackl 2000 is not actually set up to support formalization of this account. The reason is that Hackl does not countenance quantification over proper parts of a singular individual such as a cake. For relevant discussion, including analyses of entire and whole, see Morzycki 2002 and Moltmann 2005. 
Two types of class B numeral modifiers

\section{The range requirement}

Providing a general characterization of the bottom of the scale is of course just one step towards understanding the bottom-of-the-scale effect. In this section, we take the next step by exploring a possible derivation of this effect. We amend Nouwen's (2010) semantics for up to by adding an entailment that we refer to as the range requirement. We then show that this addition has other welcome consequences. In particular, we show that it derives the ignorance implications described in section $1 .{ }^{12}$

\subsection{Deriving the bottom-of-the-scale effect}

Having identified 1 and 6 as the smallest possible elements of the sets in (25) and (30), respectively, let us revisit the truth conditions in (24) and (29). According to these truth conditions, the smallest possible degree in a set of degrees, the so-called bottom of the scale, is also the maximal element of the set. Note that this is tantamount to saying that the bottom of the scale is the only element of the set in question. This means that (24) and (29), in our scenario, can be rewritten as (32) and (33), respectively.

(32) $\quad\{\mathrm{d}: \diamond \exists ! \mathrm{X}[|\mathrm{X}|=\mathrm{d} \& \llbracket$ people $\rrbracket(X)=\llbracket$ died in the $\operatorname{crash} \rrbracket(\mathrm{X})=\mathrm{T}]\}=\{1\}$

(33) $\{\mathrm{d}: \diamond \exists ! X[|X|=\mathrm{d} \& \llbracket$ eggs $\rrbracket(X)=\llbracket$ he bought $\rrbracket(X)=T]\}=\{6\}$

More generally, when up to modifies a bottom-of-the-scale numeral, the resulting truth conditions entail that the degree denoted by that numeral is the only element of the set of degrees that the modified numeral composes with.

12 While preparing the final version of this reply, we were reminded of a closely related proposal by Penka (2010). Penka applies a general semantics for superlatives to at least and at most in an attempt to compositionally derive their truth conditional contributions. By decomposing at least and at most, Penka effectively adds a range requirement (as a presupposition) to their semantics. Moreover, Penka intends for the range requirement to have the same effects regarding blocking and the derivation of ignorance implications that we discuss in sections 5.2 and 5.3 below, and analogous to our discussion in sections 6.3 and 6.4, Penka identifies the potential problems for this approach coming from weak interpretations of numerals (and other measure phrases). Naturally, however, being concerned with superlative modifiers, rather than up to, Penka does not establish a link between the range requirement and our bottom-of-the-scale effect. In fact, under Penka's proposal, we wonder how one might accomodate the observation, reported in section 4 , that this effect is absent with at most. It also appears that Penka's account inherits from Nouwen's the problems we have identified in section 3 regarding NPI licensing and the corresponding inferences. 
This fact suggests a possible way to account for the bottom-of-the-scale effect. Suppose that Nouwen's lexical entry for up to in (9) above is strengthened so as to yield the implication that the relevant set of degrees contains more than one element. Let us assume for concreteness that this implication is part of the asserted content. We then arrive at the revised lexical entry in (34), where range( $f$ ), the range requirement, expresses that (the set characterized by) f has more than one element. ${ }^{13,14}$

$$
\llbracket \text { up to } \rrbracket=\lambda \mathrm{d}_{\mathrm{d}} \cdot \lambda \mathrm{f}_{\mathrm{dt}} \cdot \max (\mathrm{f})=\mathrm{d} \& \operatorname{range}(\mathrm{f})
$$

Under our revised entry for up to, the truth conditions of (23), repeated below, are the conjunction of the equality in (32) and the range requirement in (35); likewise, the truth conditions of (28), also repeated below, are the conjunction of the equality in (33) and the range requirement in (36).

(23) [[up to] one] $\lambda \mathrm{d}[\diamond$ [[d many] people died in the crash]]

（35） $\quad \operatorname{range}(\{\mathrm{d}: \diamond \exists ! \mathrm{X}[|\mathrm{X}|=\mathrm{d} \& \llbracket$ people $\rrbracket(X)=\llbracket$ died in the $\operatorname{crash} \rrbracket(\mathrm{X})=\mathrm{T}]\})$

(28) [[up to] half a dozen] $\lambda \mathrm{d}[\diamond$ [he bought [d many] eggs]]

$$
\text { range }(\{\mathrm{d}: \diamond \exists ! X[|X|=\mathrm{d} \& \llbracket \text { eggs } \rrbracket(X)=\llbracket \text { he bought } \rrbracket(X)=T]\})
$$

The LFs in (23) and (28) are now contradictory: In their respective truth conditions, one conjunct implies that the relevant set of degrees is a singleton set, while the other implies that it is not. Under the assumption that contradictory truth conditions can be a source of unacceptability, a view recently defended in Gajewski 2003, the range requirement therefore correctly excludes (22b) and (27b) and more generally derives the bottom-of-the-scale effect.

More accurately, so far we have only shown that the particular LFs in (23) and (28) are contradictory. A complete explanation of the unacceptability of the corresponding examples will have to exclude other potential LFs for these examples as well. In particular, we will have to exclude LFs that, unlike (23) and (28), feature no possibility modal in the scope of the degree phrase.

13 It should be clear that under this revised entry, up to is still not downward entailing (in fact, it is still non-monotone), so the relevant observations in section 3 are accounted for as before.

14 In Nouwen 2008, up to is a universal quantifier over degrees in an interval. Under this view, the range requirement could be taken to follow from the general requirement that the restrictor of a universal quantifier have more than one element (see, for example, Schwarzschild 2002 for discussion). 
Two types of class B numeral modifiers

These are indeed excluded as well, as we will show in the next section. Just like in Nouwen's account, under the amendment we have proposed, LFs that feature no possibility modal in the scope of up to will not give rise to attested interpretations.

\subsection{The range requirement and blocking}

Introducing the range requirement forces us to abandon Nouwen's (2010) rationale for the obligatory presence of a silent possibility operator. We can illustrate this with (21b), repeated below.

(21b) Up to ten people died in the crash.

Recall that Nouwen's account rests on the assumption that an LF can be blocked by the availability of a semantically equivalent LF that counts as less marked. For Nouwen, the LF for (21b) given in (37) is true just in case exactly ten people died in the crash.

(37) [[up to] ten] $\lambda d[[d$ many] people died in the crash]

Nouwen's account ensures that the bare numeral example in (38a), or rather its LF in (38b), is likewise true just in case exactly ten people died in the crash and hence is equivalent to (37). Moreover, Nouwen assumes that (37) is more marked than (38b), and so the latter blocks the former.

(38) a. Ten people died in the crash.

b. [ten many] people died in the crash

It should be clear that under the revised lexical entry in (34), the LF in (37) is no longer equivalent to (38b). The addition of the range requirement renders (37) strictly stronger than (38b). Therefore, assuming that semantic equivalence is a precondition on blocking, an alternative explanation must be sought for the unavailability of (37).

Fortunately, such an alternative explanation is readily available. The LF in (37) is, in fact, not merely stronger than (38b), but actually has contradictory truth conditions. To see why, consider the set given by the degree predicate in (37), shown in (39).

$$
\{\mathrm{d}: \exists ! \mathrm{X}[|\mathrm{X}|=\mathrm{d} \& \llbracket \text { people } \rrbracket(X)=\llbracket \text { died in the } \operatorname{crash} \rrbracket(X)=\mathrm{T}]\}
$$


This set can never have more than one element: If no one died in the crash, it will be empty, and it will contain only the exact number of fatalities if someone did; so the range requirement in (40) is necessarily false.

(40) $\quad \operatorname{range}(\{\mathrm{d}: \exists ! X[|X|=\mathrm{d} \& \llbracket$ people $\rrbracket(X)=\llbracket$ died in the $\operatorname{crash} \rrbracket(X)=T]\})$

Therefore, under the assumption (already employed above) that contradictory truth conditions can keep an LF from being attested, the effect of blocking in Nouwen's account is replicated.

Moreover, the addition of a possibility operator can be shown to result in an LF whose truth conditions correspond to an observable interpretation. The reason is that a possibility operator renders an otherwise contradictory range requirement satisfiable. This will become clear in the next subsection, which examines the effect of the range requirement on attested interpretations of acceptable up to sentences. ${ }^{15}$

\subsection{The range requirement and ignorance implications}

In this section we have so far demonstrated the utility of the range requirement in preventing overgeneration by excluding problematic LFs containing bottom-of-the-scale numerals or lacking possibility modals. The range requirement was seen to exclude the LFs in question by rendering them contradictory. But surely the range requirement will have an effect on the observable interpretations derived for acceptable up to sentences, and this effect remains to be identified.

Let us therefore return to sentence (21b), repeated below. The sentence has the intended LF in (41). The truth conditions we assign to this LF are given by the conjunction of (42a) and (42b).

(21b) Up to ten people died in the crash.

(41) [[up to] ten] $\lambda \mathrm{d}[\diamond$ [[d many] people died in the crash]]

(42) a. $\max (\{\mathrm{d}: \diamond \exists ! \mathrm{X}[|\mathrm{X}|=\mathrm{d} \& \llbracket$ people $\rrbracket(X) \&$

$\llbracket$ died in the $\operatorname{crash} \rrbracket(X)=T]\})=10$

b. $\quad$ range $(\{d: \diamond \exists ! X[|X|=d \& \llbracket$ people $\rrbracket(X) \&$

$\llbracket$ died in the $\operatorname{crash} \rrbracket(X)=T]\})$

15 This account of the modal interpretation of class B modifiers is very similar to MenéndezBenito's (2010) analysis of so-called universal free choice items. 
Two types of class B numeral modifiers

The conjunct in (42a), according to which 10 is the maximal number of fatalities in the crash consistent with the speaker's evidence, is of course an entailment of the LF in (41) under Nouwen's (2010) original account as well. We accept Nouwen's assumption that this is indeed an attested entailment of sentence (21b).

Turning to (42b), let us attend to the set serving as the argument of range. This set is isolated in (25), repeated below.

(25) $\quad\{\mathrm{d}: \diamond \exists ! \mathrm{X}[|\mathrm{X}|=\mathrm{d} \& \llbracket$ people $\rrbracket(\mathrm{X})=\llbracket$ died in the $\operatorname{crash} \rrbracket(\mathrm{X})=\mathrm{T}]\}$

This set contains all non-zero degrees that could be the exact number of fatalities given the speaker's evidence. To be sure, since it is possible for this set to contain more than one element, the range requirement in (42b) can be true. This supports our conclusion at the end of the previous subsection that the addition of a possibility operator can render an otherwise contradictory LF contingent.

Moreover, saying that the set in (25) has more than one element amounts to saying that the set of worlds consistent with the speaker's evidence varies in terms of the exact number of people who died in the crash. Thus, (42b) amounts to the statement that the speaker considers it possible that there were fatalities in the crash, but does not have sufficient evidence to identify the exact number. In other words, (42b) entails speaker ignorance regarding the exact number of fatalities.

Sentence (21b) is indeed judged to imply such speaker ignorance. Of course, this is an instance of Nouwen's observation, reviewed in section 1 , that all so-called class B modifiers introduce such implications. Recall that Nouwen attributes the oddness of example (2b), repeated below, to the implausibility of speaker ignorance regarding the number of sides a hexagon has.

(2b) \#A hexagon has up to 10 sides.

Sentence (21b) illustrates the same effect, though perhaps in a less dramatic way, given that there is nothing implausible about the implied speaker ignorance regarding fatalities in the crash.

We therefore conclude that in addition to accounting for the bottom-ofthe-scale effect, the range requirement has the welcome effect of deriving attested ignorance implications contributed by up to, which under Nouwen's original proposal remained unaccounted for. 
This is a pleasing result; however, in outlining Nouwen's analysis of upperbound class B modifiers in section 2, we have permitted ourselves a slight departure from his actual account. While inconsequential for Nouwen's analysis of numeral modifiers, this departure raises questions for our modification of it. This is the topic of the next section.

\section{Weak many}

We reported that Nouwen (2010) posits a counting quantifier, many, that combines with numerals to form degree phrases. Actually, however, Nouwen assumes that there are two such counting quantifiers (see footnote 2). In addition to the many in (5), repeated below, Nouwen also posits a second many, given in (43), which we write as many $y_{w}$.

$$
\begin{aligned}
& \llbracket \text { many } \rrbracket=\lambda d_{d} \cdot \lambda f_{e t} \cdot \lambda g_{e t} \cdot \exists ! X[|X|=d \& f(X)=g(X)=T] \\
& \llbracket \text { many }_{\mathrm{w}} \rrbracket=\lambda d_{d} \cdot \lambda f_{e t} \cdot \lambda g_{e t} \cdot \exists X[|X|=d \& f(X)=g(X)=T]
\end{aligned}
$$

The subscript $w$ is meant to signal that the many $y_{w}$ in (43) derives truth conditions that are weaker than those derived by the strong many in (5). Consider again example (3) and the LF in (4), repeated below. We have seen that (4) is true just in case exactly three students attended. In contrast, (43) ensures that (44) is true just as long as no fewer than three students attended.

(3) Three students attended.

(4) [[three many] students] attended

(44) [[three many $\left.{ }_{w}\right]$ students] attended

Postulating many in $_{\text {(43) }}$ is motivated by the well-known observation that sentences with bare numerals indeed allow for such weak interpretations (see Geurts 2006 and the references provided therein). In this section, we describe the consequences of introducing many $y_{w}$ for Nouwen's original account as well as for our own modification of it. We show that it is innocuous for the former, but not for the latter. Specifically, we show that while our account of the bottom-of-the-scale effect is preserved, our derivation of ignorance implications is not. Note that we will not solve this problem. The purpose of this section is merely to identify the price to pay for the kind of modification to Nouwen's account that we have proposed. 
Two types of class B numeral modifiers

\subsection{Nouwen's account revisited}

Nouwen (2010) carefully demonstrates that when the two counting quantifiers combine with upper-bound class B modifiers, the semantic difference between the strong version in (5) and the weak version in (43) is systematically neutralized. It will be useful to review the reason for this neutralization by attending again to (21b), repeated below. Consider first the LF with many in (37), repeated below, and its counterpart with many $w_{w}$ in (45).

(21b) Up to ten people died in the crash.

(37) [[up to] ten] $\lambda d[$ [d many] people died in the crash]

(45) [[up to] ten] $\lambda d\left[\left[d\right.\right.$ many $\left._{w}\right]$ people died in the crash]

Consider the sets denoted by the derived degree predicates in (37) and (45), shown in (39) (repeated from earlier) and (46).

(39) $\{\mathrm{d}: \exists ! \mathrm{X}[|\mathrm{X}|=\mathrm{d} \& \llbracket$ people $\rrbracket(\mathrm{X})=\llbracket$ died in the $\operatorname{crash} \rrbracket(\mathrm{X})=\mathrm{T}]\}$

(46) $\{\mathrm{d}: \exists \mathrm{X}[|\mathrm{X}|=\mathrm{d} \& \llbracket$ people $\rrbracket(\mathrm{X})=\llbracket$ died in the $\operatorname{crash} \rrbracket(\mathrm{X})=\mathrm{T}]\}$

Suppose that there have been fatalities in the crash and let $\mathrm{f}$ be their exact number. We have seen that (39) is \{f\}. In contrast, (46) is the interval [1, f], which apart from $\mathrm{f}$ includes all lower cardinalities down to the bottom of the scale, 1. This implies that the two sets in (39) and (46) necessarily have the same maximum. Accordingly, the two statements in (47) and (48) are equivalent, for any cardinality $\mathrm{n}$, and therefore under Nouwen's semantics for up to, the LFs in (37) and (45) have identical truth conditions.

(47) $\max (\{\mathrm{d}: \exists ! \mathrm{X}[|\mathrm{X}|=\mathrm{d} \& \llbracket$ people $\rrbracket(X)=\llbracket$ died in the $\operatorname{crash} \rrbracket(\mathrm{X})=\mathrm{T}]\})=\mathrm{n}$ (48) $\max (\{\mathrm{d}: \exists X[|X|=\mathrm{d} \& \llbracket$ people $\rrbracket(X)=\llbracket$ died in the $\operatorname{crash} \rrbracket(X)=\mathrm{T}]\})=\mathrm{n}$

The same is true for the LFs in (41) (repeated from earlier) and (49), where the silent possibility modal has been added to the scope of up to.

(41) [[up to] ten] $\lambda \mathrm{d}[\diamond$ [[d many] people died in the crash]]

(49) $\quad\left[\right.$ up to] ten] $\lambda d\left[\nabla\left[\left[d\right.\right.\right.$ many $\left._{\mathrm{w}}\right]$ people died in the crash $\left.]\right]$

Now the derived degree predicates denote the sets in (25) (repeated from earlier) and (50).

(25) $\quad\{\mathrm{d}: \diamond \exists ! \mathrm{X}[|\mathrm{X}|=\mathrm{d} \& \llbracket$ people $\rrbracket(\mathrm{X})=\llbracket$ died in the $\operatorname{crash} \rrbracket(\mathrm{X})=\mathrm{T}]\}$ 
(50) $\quad\{\mathrm{d}: \diamond \exists \mathrm{X}[|\mathrm{X}|=\mathrm{d} \& \llbracket$ people $\rrbracket(\mathrm{X})=\llbracket$ died in the $\operatorname{crash} \rrbracket(\mathrm{X})=\mathrm{T}]\}$

Suppose that according to the speaker's evidence, someone may have died in the crash and let $\mathrm{p}$ be the largest number of fatalities consistent with that evidence. ${ }^{16}$ Then (25) is a set whose maximal element is p and (50) is the set $[1, p]$. This entails once again that the sets in (25) and (50) have the same maximum element. For any cardinality n, the statements in (51) and (52) are equivalent, and so (41) and (49) have identical truth conditions.

(51) $\quad \max (\{\mathrm{d}: \diamond \exists ! \mathrm{X}[|\mathrm{X}|=\mathrm{d} \& \llbracket$ people $\rrbracket(X) \& \llbracket$ died in the $\operatorname{crash} \rrbracket(\mathrm{X})=\mathrm{T}]\})$

(52) $\max (\{\mathrm{d}: \diamond \exists \mathrm{X}[|\mathrm{X}|=\mathrm{d} \& \llbracket$ people $\rrbracket(\mathrm{X}) \& \llbracket$ died in the $\operatorname{crash} \rrbracket(\mathrm{X})=\mathrm{T}]\})$

The addition of many $_{w}$, then, is innocuous for Nouwen's original account of upper-bound class B modifiers. Unfortunately, this welcome feature is not shared by the revised version we have presented above. While our account of the bottom-of-the-scale effect is preserved under the addition of many $y_{w}$, our rationale for why up to must be accompanied by a possibility operator must be revised, and our proposed derivation of ignorance implications is lost. We dedicate the following three subsections to substantiating these points.

To facilitate the presentation, though, it will be useful to first highlight another pair of equivalencies. Recall that the sets in (46) and (50), the sets arising from LFs with many $y_{w}$, necessarily denote intervals of the form $[1, \mathrm{n}]$, sets consisting of a maximum $\mathrm{n}$ and all smaller cardinalities down to the bottom of the scale, 1 . This means that the membership of these sets is fully determined by their maximal elements: For any cardinality n, the maximality statements in (48) and (52) are equivalent to the equalities in (53) and (54).

(54) $\{\mathrm{d}: \diamond \exists \mathrm{X}[|\mathrm{X}|=\mathrm{d} \& \llbracket$ people $\rrbracket(\mathrm{X})=\llbracket$ died in the $\operatorname{crash} \rrbracket(\mathrm{X})=\mathrm{T}]\}=[1, \mathrm{n}]$

Having a name for these two equivalencies will simplify the exposition below, so we will collectively refer to them as the range law.

16 The sets in (25) and (50), even if non-empty, are not guaranteed to have maximal elements. However, we can safely focus on the case where they do, as this is a presupposition triggered by up to in Nouwen's account. 
Two types of class B numeral modifiers

\subsection{Bottom-of-the-scale effect revisited}

Above we proposed that (22b), repeated below, is excluded because it only has a contradictory interpretation. To maintain this account, we now have to consider the LF with many $y_{w}$ in (55). We want to show that this LF shares the contradictory truth conditions of its counterpart with (strong) many (see (23) in section 5.1).

(22b) \#Up to one person died in the crash.

(55) [[up to] one] $\lambda \mathrm{d}\left[\diamond\left[\left[\mathrm{d}^{-}\right.\right.\right.$any $\left.\mathrm{w}_{\mathrm{w}}\right]$ people died in the crash]]

Given the semantics of many ${ }_{w}$, the truth conditions we assign to (55) are given by the conjunction of (56a) and (56b).
a. $\max (\{\mathrm{d}: \diamond \exists X[|\mathrm{X}|=\mathrm{d} \& \llbracket$ people $\rrbracket(X)=$ $\llbracket$ died in the $\operatorname{crash} \rrbracket(X)=T]\})=1$
b. $\quad$ range $(\{d: \diamond \exists X[|X|=d \& \llbracket$ people $\rrbracket(X)=$
$\llbracket$ died in the $\operatorname{crash} \rrbracket(X)=T]\})$

Just as we saw in section 5.1 with (strong) many, a set of degrees whose maximum is the bottom of the scale is a singleton set containing that degree. It follows that (56a) is equivalent to (57). (This is also evident from the range law since $[1,1]=\{1\}$.)

(57) $\{\mathrm{d}: \diamond \exists X[|\mathrm{X}|=\mathrm{d} \& \llbracket$ people $\rrbracket(\mathrm{X}) \llbracket$ died in the $\operatorname{crash} \rrbracket(\mathrm{X})=\mathrm{T}]\}=\{1\}$

Therefore the conjunction of (56a) and (56b) is inconsistent, hence the LF in (55) is excluded in the same way as its variant with strong many in (23).

It should moreover be clear that omitting the possibility operator from (55) does not change the relevant semantic relations: The maximum of the relevant set is still the bottom of the scale (and the range law will apply as before), and so a contradiction will again be derived. The addition of many, then, does not save bottom-of-the-scale numerals from yielding contradictory truth conditions, and so we conclude that our account of the bottom-of-thescale effect still applies correctly.

\subsection{Blocking revisited}

Nouwen (2010) proposes that unless they contain a possibility modal (in the immediate scope of the degree phrase), LFs with upper-bound class B 
modifiers are blocked by less-marked LFs with bare numerals. In our revised account, only positing strong many, we proposed that instead of being blocked by markedness, the relevant LFs are excluded by virtue of being contradictory. Having added many $y_{w}$, we now have to revisit this account. We will focus here on numerals above the bottom of the scale, since we just discussed how bottom-of-the-scale numerals give rise to contradictions.

Consider again example (21b), repeated below. We need to examine the predicted interpretation of the LF in (45), repeated below, which features many $y_{w}$ but no possibility modal.

(21b) Up to ten people died in the crash.

(45) [[up to] ten] $\lambda d\left[\left[d\right.\right.$ many $\left._{w}\right]$ people died in the crash]

Under our semantics for up to, the truth conditions of (45) are the conjunction of (58a) and (58b).

$$
\begin{array}{r}
\text { a. } \quad \begin{array}{r}
\max (\{\mathrm{d}: \exists \mathrm{X}[|\mathrm{X}|=\mathrm{d} \& \llbracket \text { people } \rrbracket(X)=\llbracket \text { died in the } \operatorname{crash} \rrbracket(\mathrm{X})=\mathrm{T}]\}) \\
=10
\end{array} \\
\text { b. } \quad \operatorname{range}(\{\mathrm{d}: \exists \mathrm{X}[|\mathrm{X}|=\mathrm{d} \& \llbracket \text { people } \rrbracket(\mathrm{X})=\llbracket \text { died in the } \operatorname{crash} \rrbracket(\mathrm{X})=\mathrm{T}]\})
\end{array}
$$

Note that according to the range law, (58a) is equivalent to (59). Since in this case the numeral is above the bottom of the scale, the set of cardinalities it determines according to the range law is necessarily a range.

$$
\{\mathrm{d}: \exists \mathrm{X}[|\mathrm{X}|=\mathrm{d} \& \llbracket \text { people } \rrbracket(\mathrm{X})=\llbracket \text { died in the } \operatorname{crash} \rrbracket(\mathrm{X})=\mathrm{T}]\}=[1,10]
$$

Accordingly, the maximality statement in (58a) entails the range requirement in (58b). It follows that the conjunct in (58b) is effectively redundant: The truth conditions of (45) are given by (58a) alone.

So our revised semantics for up to assigns to (45) the very same truth conditions as Nouwen's original semantics: The LF is true just in case exactly ten people died in the crash. Since these truth conditions are contingent, our rationale for why up to requires a possibility operator in its scope is not preserved under the addition of many.

As things stand, then, we are in danger of predicting an unattested interpretation of (21b), according to which exactly ten people died in the crash. Evidently, we must abandon the idea that the presence of a possibility operator is always motivated by the need to prevent contradiction.

Fortunately, though, given that we assign to (45) the very same truth conditions as Nouwen, a natural modification suggests itself. To force the 
Two types of class B numeral modifiers

presence of a possibility operator in an LF with many $_{w}$, we can adopt Nouwen's notion of blocking by markedness, which after all is not inconsistent with anything we have proposed. So, following Nouwen, we could assume that (45) is excluded by virtue of being more marked than the equivalent LF in (38b), repeated from earlier.

(38b) [[ten many] people died in the crash]

In the resulting account, then, there would be two different reasons why up to requires the presence of a possibility operator (with numerals above the bottom of the scale): In LFs with strong many, the possibility operator renders an otherwise contradictory LF contingent; in LFs with many $y_{w}$, the possibility operator prevents the LF from being blocked by a less-marked competitor.

\subsection{Ignorance implications revisited}

We have proposed to credit ignorance implications to the range requirement introduced by the lexical semantics of up to. Once again, we need to go back and examine the effect of the addition of $m a n y_{w}$. Let us return to the LF in (49), repeated below.

(49) [[up to] ten] $\lambda \mathrm{d}\left[\diamond\left[\left[\mathrm{d}^{\mathrm{many}} \mathrm{w}_{\mathrm{w}}\right]\right.\right.$ people died in the crash]]

Under our account, the truth conditions of (49) are given by the conjunction of (6oa) and (6ob). According to the range law, we moreover have the equivalence of (6oa) and (61).

$$
\begin{gathered}
\text { a. } \max (\{\mathrm{d}: \diamond \exists X[|\mathrm{X}|=\mathrm{d} \& \llbracket \text { people } \rrbracket(X)= \\
\text { b. } \quad \operatorname{range}(\{\mathrm{d}: \diamond \exists X[|\mathrm{X}|=\mathrm{d} \& \llbracket \text { peopled in the } \operatorname{crash} \rrbracket(X)=\mathrm{X}]\})=10 \\
\llbracket \text { died in the } \operatorname{crash} \rrbracket(X)=\mathrm{T}]\})
\end{gathered}
$$

(61) $\quad\{\mathrm{d}: \diamond \exists X[|\mathrm{X}|=\mathrm{d} \& \llbracket$ people $\rrbracket(X)=\llbracket$ died in the $\operatorname{crash} \rrbracket(X)=\mathrm{T}]\}=[1,10]$

This means that much like in the case studied in the previous subsection, the range requirement ends up being redundant, as it is entailed by (6oa). ${ }^{17}$ So in

17 Despite initial appearance, the statement in (61) does not imply speaker ignorance. Specifically, it is consistent with the speaker being certain that exactly ten people died in the crash. So (61) does not entail that the set of worlds consistent with the speaker's evidence varies in terms of exact number of fatalities. In contrast, equating the set in (25) with a range like 
this case, too, our semantics for up to derives the very same truth conditions as Nouwen's.

This is certainly not a welcome result. The range requirement being redundant, our account of ignorance implications is lost. We showed that the range requirement derives ignorance implications for LFs with strong many. However, if we assume that many ${ }_{w}$ is freely available as well, we admit LFs where the range requirement does not actually strengthen the truth conditions in any way, and so we no longer predict that up to is perceived to always introduce these implications.

So if one admits many $_{w}$, an alternative derivation of ignorance implications needs to be sought. But insisting on deriving ignorance implications through the range requirement, by excluding many $_{w}$, has its price as well. As reported above, many $y_{w}$ is motivated by weak readings of bare numerals, so an alternative account of those would be needed. ${ }^{18}$

\section{Summary and conclusion}

What we consider the main result of this commentary is that so-called upperbound class B numeral modifiers come in two types. This is shown, first, by NPI licensing facts and intuitions on corresponding inferences. The class B numeral modifier at most licenses NPIs and is downward entailing, according to intuitions on inferences. In contrast, the class B modifier up to has neither of these properties. Second, while at most can combine with bottom-of-the-scale numerals, up to cannot. These basic semantic contrasts suffice to establish that at most and up to cannot share the same semantic interpretation.

More specifically, NPI licensing data and the corresponding inferences demonstrate that Nouwen's (2010) semantics for upper-bound class B modifiers is not correct for at most. In attempting to apply it to up to, we identified

$[1,10]$ does imply speaker ignorance.

18 The literature provides possible starting points for either direction of analysis. One might hope to derive ignorance implications as conversational implicatures, perhaps along the lines of Büring 2008, which provides an analysis of at least (see also Cummins \& Katsos 2010 and Schwarz \& Shimoyama 2011). Conversely, one might follow Kratzer 2003 in employing situation semantics to derive apparent weak readings from strong lexical meanings, such as the strong many we have been discussing. A third possibility is that many $y_{w}$ is an available lexical item with a suitably limited distribution: Specifically, it cannot combine with a trace of a numeral modified by up to; unfortunately, however, we don't see what principle of grammar such a restriction might follow from. 
Two types of class B numeral modifiers

two shortcomings. First, the analysis fails to derive the bottom-of-the-scale effect; second, it surprisingly fails to derive the very ignorance implications that Nouwen identified as a defining feature of class B modifiers.

We presented an amendment of Nouwen's account that adds the range requirement to the truth conditional content of up to. This amendment captures the bottom-of-the-scale effect. Under the additional assumption that the counting quantifier many is always strong, but not without this assumption, we also derive ignorance implications. So the extent to which our proposal improves upon Nouwen's original account depends on whether a weak counting quantifier is assumed to be available.

Apart from the issue raised by $\operatorname{many}_{w}$, one additional concern about our proposed derivation of ignorance implications with up to is that it might be insufficiently general, as it is unclear how it would carry over to at most. However, in view of the semantic differences between up to and at most that we have identified, it is not clear to us that the ignorance implications associated with the two expressions should even have the same source. Thus, our discussion raises the possibility that there are two types of ignorance implications, and hence that this common feature of class B numeral modifiers is not necessarily indicative of a shared analysis.

To be sure, there may well be subclasses of class B modifiers which do form semantic and pragmatic natural classes. If morphology is a guide, we expect at least to pattern with at most (see Penka 2010). But there are other numeral modifiers characterized as class B by Nouwen, such as minimally and maximally, whose place in the typology remains to be determined.

\section{References}

Büring, Daniel. 2008. The least at least can do. Proceedings of the 26th West Coast Conference on Formal Linguistics (WCCFL). 114-120. http: //www.lingref.com/cpp/wccfl/26/paper1662.pdf.

Chierchia, Gennaro \& Sally McConnell-Ginet. 2000. Meaning and grammar: an introduction to semantics, 2nd edn. Cambridge, MA: MIT Press.

Cummins, Chris \& Napoleon Katsos. 2010. Comparative and superlative quantifiers: pragmatic effects of comparison type. Journal of Semantics 27(3). 271-305. http://dx.doi.org/10.1093/jos/ffqoo6.

Gajewski, Jon. 2003. On analyticity in natural language. Manuscript. MIT, Cambridge. http://gajewski.uconn.edu/papers/analytic.pdf. 
Bernhard Schwarz, Brian Buccola, and Michael Hamilton

Gajewski, Jon. 2008. NPI any and connected exceptive phrases. Natural Language Semantics 16(1). 69-110. http://dx.doi.org/10.1007/s11050-0079025-8.

Geurts, Bart. 2006. Take 'five': the meaning and use of a number word. In Svetlana Vogeleer \& Liliane Tasmowski (eds.), Non-definiteness and plurality, 311-329. Amsterdam \& Philadelphia: John Benjamins Publishing. Hackl, Martin. 200o. Comparative quantifiers. Massachusetts Institute of Technology PhD thesis. http://dx.doi.org/1721.1/8765.

Kiparsky, Paul. 1973. "Elsewhere" in phonology. In Stephen R. Anderson \& Paul Kiparsky (eds.), A festschrift for Morris Halle, 93-106. New York: Holt, Reinhart \& Winston.

Kiparsky, Paul. 1983. Word formation and the lexicon. Proceedings of the 1982 Mid-America Linguistics Conference (MALC). 3-29.

Kratzer, Angelika. 2003. Scalar implicatures: are there any? Talk given at Workshop on Polarity, Scalar Phenomena, and Implicatures. University of Milan-Bicocca, June 18, 2003. http://semanticsarchive.net/Archive/ GJiYjQxN/.

Krifka, Manfred. 2007. More on the difference between more than two and at least three. http://amor.cms.hu-berlin.de/ h2816i3x/Talks/SantaCruz20o7. pdf.

Ladusaw, William A. 1979. Polarity sensitivity as inherent scope relations. University of Texas at Austin PhD thesis.

Menéndez-Benito, Paula. 2010. On universal Free Choice items. Natural Language Semantics 18(1). 33-64. http://dx.doi.org/10.1007/s11050-0099050-X.

Moltmann, Friederike. 2005. Part structures in situations: the semantics of individual and whole. Linguistics and Philosophy 28(5). 599-641. http: //dx.doi.org/10.1007/s10988-005-2142-2.

Morzycki, Marcin. 2002. Wholes and their covers. Proceedings of the 12th Semantics and Linguistic Theory conference (SALT XII). 184-203. http: //elanguage.net/journals/index.php/salt/article/view/12.184/1781.

Nouwen, Rick. 2008. Directionality in numeral quantifiers: the case of up to. Proceedings of the 18th Semantics and Linguistic Theory conference (SALT XVIII). 569-582. http://elanguage.net/journals/index.php/salt/article/ view/18.569/1919.

Nouwen, Rick. 2010. Two kinds of modified numerals. Semantics \& Pragmatics 3(3). 1-41. http://dx.doi.org/10.3765/sp.3.3. 
Two types of class B numeral modifiers

Penka, Doris. 2010. A superlative analysis of superlative scalar modifiers. Paper presented at Sinn und Bedeutung $(\mathrm{SuB})$ 15. Universität des Saarlandes, September 9-11, 2010. http://www.uni-saarland.de/fak4/fr41/reich/conf/ sub15/program/abstracts/penka-sub15.pdf.

Schwarz, Bernhard \& Junko Shimoyama. 2011. Negative islands and obviation by wa in Japanese degree questions. Proceedings of the 2oth Semantics and Linguistic Theory conference (SALT XX). 702-719. http://elanguage. net/journals/index.php/salt/article/view/20.702/1465.

Schwarzschild, Roger. 2002. Singleton indefinites. Journal of Semantics 19(3). 289-314. http://dx.doi.org/10.1093/jos/19.3.289.

Bernhard Schwarz

Department of Linguistics

1085 Dr. Penfield

Montréal, Québec

$\mathrm{H}_{3} \mathrm{~A} 1 \mathrm{~A} 7$

Canada

bernhard.schwarz@mcgill.ca

Michael Hamilton

Department of Linguistics

1085 Dr. Penfield

Montréal, Québec

$\mathrm{H}_{3} \mathrm{~A}{ }_{1} \mathrm{~A} 7$

Canada

michael.hamilton@mail.mcgill.ca
Brian Buccola

Department of Linguistics

1085 Dr. Penfield

Montréal, Québec

$\mathrm{H}_{3} \mathrm{~A}{ }_{1} \mathrm{~A} 7$

Canada

brian.buccola@mail.mcgill.ca 